\title{
Complex Vector Model of the Squirrel-Cage Induction Machine Including Instantaneous Rotor Bar Currents
}

\author{
Alfredo R. Muñoz and Thomas A. Lipo, Fellow, IEEE
}

\begin{abstract}
In this paper, a new detailed mathematical derivation of the squirrel-cage induction machine $d-q$ model is introduced. The model is based on coupled magnetic circuit theory and complex space-vector notation and takes into account the actual nonsinusoidal rotor bar distribution. It is shown for the first time that, given the structural symmetry of the induction machine, both stator and rotor circuits can be modeled by the simple set of only four coupled differential equations, i.e., the $d-q$ model. More importantly, the number of equations does not depend on the number of rotor bars, and the model is valid even if the number of bars per pole is not an integer number. This enormous simplification is achieved without loss of generality nor loss of any information contained in the full set of equations, and it is valid for any operating condition. The actual $n$ rotor bars and end-ring currents are fully included in the model, and they are obtained directly by using a simple vector transformation. In addition, the three-phase rotor equivalent parameters are obtained. Secondorder effects, such as skin effect in the rotor bars, can be taken into account by simply modifying the bar and end-ring resistance values. An equivalent circuit based on the model is also derived.
\end{abstract}

Index Terms-Complex vector, $d-q$ model, induction machine, modeling, rotor bar, rotor current, simulation, squirrel cage.

\section{INTRODUCTION}

$\mathbf{T}$ HE well-known $d-q$ model of induction machines is based on the assumption that both stator and rotor windings are sinusoidally distributed in space. Although the rotor cage is clearly nonsinusoidally distributed, it is claimed that it can be replaced by an equivalent distributed winding [1]-[3]. A formal derivation of such an equivalence, however, is rarely found in the literature, and in those few cases where a more formal approach has been adopted, the derivation becomes very complex, requiring simplifying assumptions [4], [5].

In the past, several authors have used the coupled magnetic circuit theory to model a squirrel-cage induction machine [6]-[12]. The method consists of describing the machine as a set of multiple coupled circuits defined by self- and mutual-

Paper IPCSD 98-78, presented at the 1998 Industry Applications Society Annual Meeting, St. Louis, MO, October 12-16, and approved for publication in the IEEE TRANSACTIONS ON INDUSTRY APPLICATIONS by the Electric Machines Committee of the IEEE Industry Applications Society. Manuscript released for publication November 30, 1998.

A. R. Muñoz is with the Department of Electrical and Computer Engineering, University of Wisconsin, Madison, WI 53706-1691, USA, on leave from the Electrical Engineering Department, Technical University Santa María, Valparaíso, Chile (e-mail: alfredo@cae.wisc.edu).

T. A. Lipo is with the Department of Electrical and Computer Engineering, University of Wisconsin, Madison, WI 53706-1691, USA (e-mail: lipo@engr.wisc.edu).

Publisher Item Identifier S 0093-9994(99)07496-4. inductance matrices. The resultant set of differential equations is then solved for each rotor position.

In general, for a machine having $n$ rotor bars, this approach leads to a model having $n+3$ nonlinear simultaneous differential equations (plus the mechanical equation), requiring huge computational power [6], [7]. Although the number of equations can be somewhat reduced when there is an integer number of rotor bars per pole, the task of solving such a system of differential equations remains a formidable one.

In this paper, it is shown that by using a particular spacevector transformation, the machine can be fully modeled using only four coupled differential equations. This enormous simplification is achieved without loss of generality nor loss of any information contained in the full set of equations, and it is valid for any operating condition. More importantly, the number of equations does not depend on the number of rotor bars, and it is valid even if the number of bars per pole is not an integer number. The model predicts the three-phase rotor equivalent parameters in terms of the actual rotor bar resistance and inductance values, as well as giving the actual rotor bar and end-ring currents. Since the model uses the actual rotor bar and end-ring parameters, second-order effects, such as skin effect, can be easily incorporated.

\section{STATOR MODEL}

The coupled magnetic circuit theory and complex spacevector representation will be used throughout the derivation. This technique is chosen because of the great deal of simplification that can be achieved, as well as its generality. The following general assumptions are made:

- negligible saturation;

- uniform air gap;

- stator windings sinusoidally distributed;

- negligible interbar current.

Although a sinusoidally distributed stator winding is assumed, other winding distributions could also be analyzed by simply using superposition. This approach is justified by the well-known fact that different space harmonic components do not interact [12].

The total stator flux vector $\boldsymbol{\Lambda}_{s}$ can be separated into a part due to the stator currents and one due to the mutual coupling with the rotor circuit. For clarity of explanation, each term will be developed separately. 


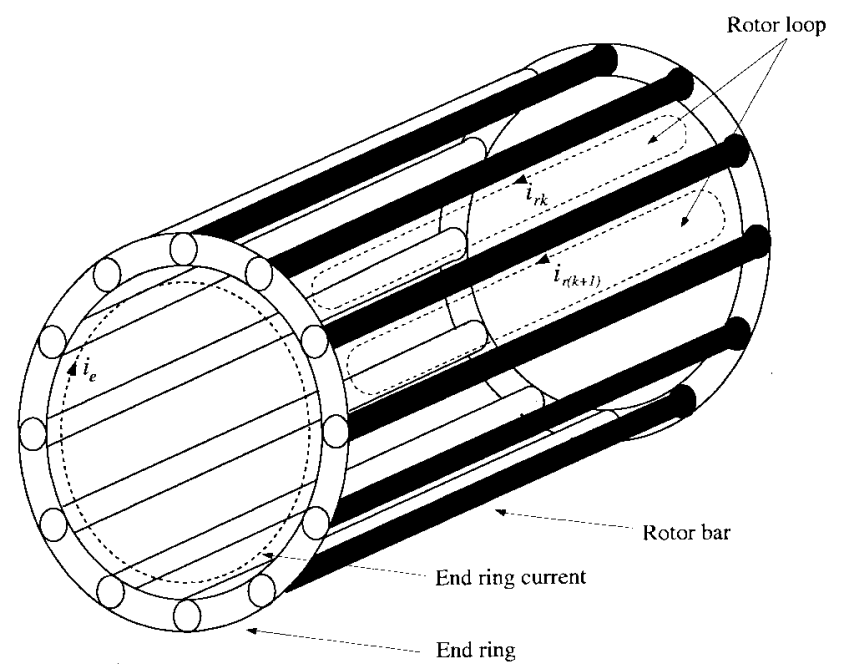

Fig. 1. Elementary rotor loops and current definitions.

\section{A. Stator Flux due to Stator Currents}

For sinusoidally distributed windings, the stator flux due to the stator currents $\boldsymbol{\Lambda}_{s s}$ is known to be [1]

$$
\left[\begin{array}{l}
\lambda_{a s s} \\
\lambda_{b s s} \\
\lambda_{c s s}
\end{array}\right]=\left[\begin{array}{ccc}
L_{l s}+L_{m s} & -\frac{L_{m s}}{2} & -\frac{L_{m s}}{2} \\
-\frac{L_{m s}}{2} & L_{l s}+L_{m s} & -\frac{L_{m s}}{2} \\
-\frac{L_{m s}}{2} & -\frac{L_{m s}}{2} & L_{l s}+L_{m s}
\end{array}\right] \mathbf{i}_{\mathbf{s}}
$$

where $L_{l s}$ and $L_{m s}$ are the leakage and magnetizing inductances of the stator windings. Applying the three-phase space-vector definition to (1) yields

$$
\underline{\lambda}_{s s}=\frac{2}{3}\left(\lambda_{a s s}+\underline{a} \lambda_{b s s}+\underline{a}^{2} \lambda_{c s s}\right)
$$

where $\underline{a}$ is the complex number $e^{j(2 \pi / 3)}$ and $\underline{\lambda}_{s s}$ is the complex space vector, thus

$$
\underline{\lambda}_{s s}=\left(L_{l s}+\frac{3}{2} L_{m s}\right) \underline{\mathbf{i}}_{s}
$$

where

$$
\underline{\mathbf{i}}_{s}=\frac{2}{3}\left(i_{a s}+\underline{a} i_{b s}+\underline{a}^{2} i_{c s}\right)
$$

is the space-vector representation of the stator current.

The magnetizing inductance $L_{m s}$ for a winding having $N_{s}$ turns per phase is given by [1]

$$
L_{m s}=\frac{\mu_{o} l r}{g} N_{s}^{2}\left(\frac{\pi}{4}\right)
$$

where $l$ is the stack length, $r$ the mean air-gap radius and $g$ the air-gap length.

\section{B. Stator Flux Due to Rotor Currents}

The rotor cage is normally modeled as $n$ identical and equally spaced loops [4], [5], [8]. As shown in Fig. 1, each loop is formed by two adjacent rotor bars and the connecting portions of the end rings between them. Each loop is magnetically coupled to all the other loops (circuits) and to all three stator phases.

The stator flux linkage due to the rotor currents is

$$
\boldsymbol{\Lambda}_{s r}=\left[\begin{array}{c}
\lambda_{a s r} \\
\lambda_{b s r} \\
\lambda_{c s r}
\end{array}\right]=\left[\begin{array}{llll}
L_{a 1} & L_{a 2} & \cdots & L_{a n} \\
L_{b 1} & L_{b 2} & \cdots & L_{b n} \\
L_{c 1} & L_{c 2} & \cdots & L_{c n}
\end{array}\right]\left[\begin{array}{c}
i_{r 1} \\
\vdots \\
i_{r n}
\end{array}\right]
$$

where $L_{x i}$ represents the mutual inductance between the stator phase $x$ and the rotor loop $i$. Neglecting the MMF drop in the iron, these inductances are conveniently calculated by means of winding functions [13], [14]. According to this theory the mutual inductance between two arbitrary machine windings " $i$ " and " $j "$ is given by

$$
L_{i j}(\theta)=\mu_{o} l r \int_{0}^{2 \pi} g^{-1}(\varphi, \theta) N_{i}(\varphi, \theta) N_{j}(\varphi, \theta) d \varphi
$$

where $\theta$ is the angular rotor position with respect to some arbitrary reference, $\varphi$ is a particular point along the air gap, $g^{-1}(\varphi, \theta)$ is the inverse air-gap function, and $N(\varphi, \theta)$ is the winding function defined as the spatial distribution of MMF due to a unit current flowing in the winding. If the air gap is assumed to be uniform and small compared to the rotor radius, the inverse air-gap function becomes simply a constant $g^{-1}$, and it can be taken out of the integral. In this case, to find the mutual inductance, we only need to define the winding functions.

Consider the stator windings and rotor bar disposition shown in Fig. 2. Taking as a reference the magnetic axis of phase $a$, the normalized stator winding function for this phase is [14]

$$
N_{a}(\theta)=\frac{N_{s}}{2} \cos (\theta)
$$

The normalized winding functions of phases $b$ and $c$ are simply displaced by $\pm 120^{\circ}$ with respect to $N_{a}$.

The normalized winding function for the $i$ th rotor loop, given by the MMF distribution produced by $1 \mathrm{~A}$ of current flowing through the $i$ th loop, is shown in Fig. 2. For all the other loops, the normalized winding functions are similar, changing only the relative phase angle as defined by the position of each loop with respect to the reference point. Mathematically, this function is defined by

$$
N_{i}(\theta)= \begin{cases}-\alpha_{r} / 2 \pi, & 0<\theta \leq \theta_{i} \\ 1-\alpha_{r} / 2 \pi, & \theta_{i}<\theta \leq \theta_{i+1} \\ -\alpha_{r} / 2 \pi, & \theta_{i+1}<\theta \leq 2 \pi\end{cases}
$$

where $\alpha_{r}$ is the angle between two adjacent rotor bars and $\theta_{i}$ and $\theta_{i+1}$ define the position of the bars forming the loop.

The mutual inductance between phase $a$ and the $i$ th rotor loop is

$$
\begin{aligned}
L_{a i} & =\frac{\mu_{o} l r}{g} \int_{0}^{2 \pi} N_{a}(\theta) N_{i}(\theta) d \theta \\
& =\frac{\mu_{o} l r}{g} \frac{N_{s}}{2}\left[\sin \alpha_{r} \cos \theta_{i}-\left(1-\cos \alpha_{r}\right) \sin \theta_{i}\right] .
\end{aligned}
$$




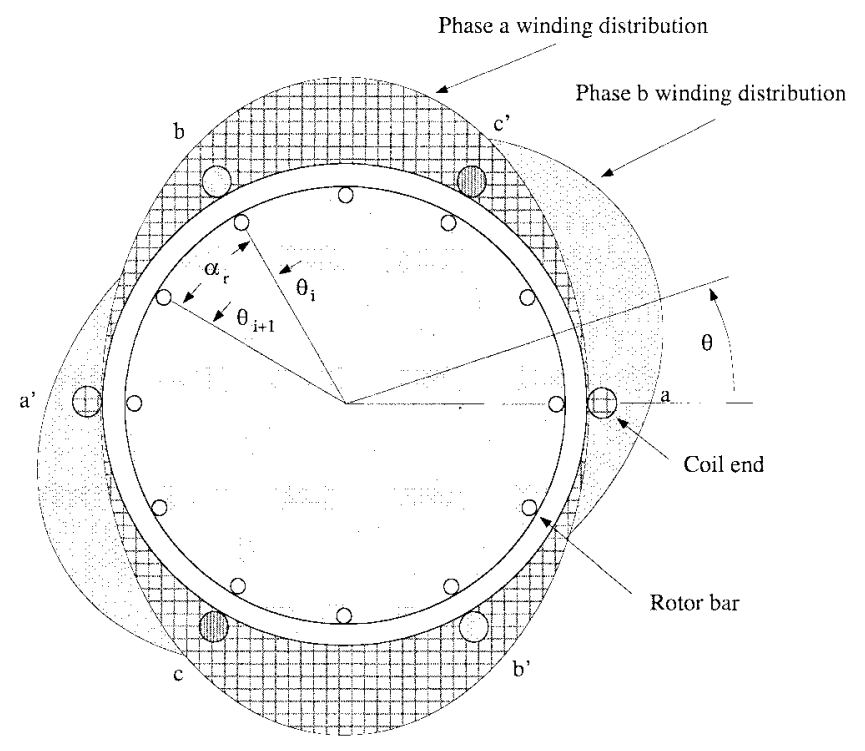

(a)

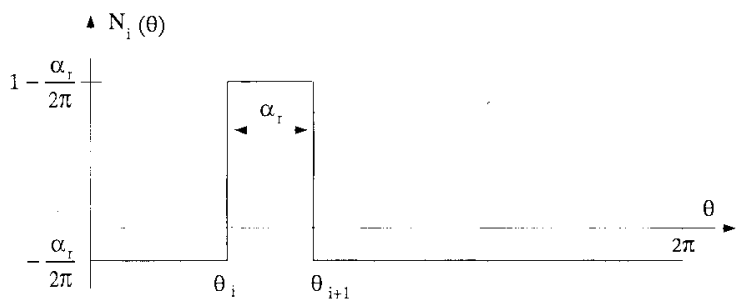

(b)

Fig. 2. (a) Stator windings distribution. (b) Rotor loop winding function.

Since each rotor loop is symmetrically located along the rotor periphery, $\theta_{i}$ can be expressed in terms of an arbitrary rotor angle $\theta_{r}$ and the angle between adjacent rotor bars $\alpha_{r}$ as

$$
\theta_{i}=\theta_{r}+(i-1) \alpha_{r} .
$$

By using trigonometric identities, (10) can be written as

$$
L_{a i}\left(\theta_{i}\right)=L_{m} \cos \left(\theta_{r}+(i-1) \alpha_{r}+\delta\right)
$$

where

$$
L_{m}=\frac{4}{\pi} \frac{\sin (\delta)}{N_{s}} L_{m s}
$$

and

$$
\delta=\frac{\alpha_{r}}{2} .
$$

The mutual inductance of the $i$ th rotor loop with respect to phases $b$ and $c$ are found by phase shifting $L_{a i}$ by $\pm 120^{\circ}$.

Using the Euler relation and substituting the mutual inductance expressions into (6) yields

$$
\begin{gathered}
{\left[\begin{array}{c}
\lambda_{a s r} \\
\underline{a}_{b s r} \\
\underline{a}^{2} \lambda_{c s r}
\end{array}\right]=} \\
+e^{-j\left(\theta_{r}+\delta\right)}\left[\begin{array}{cccc}
1 & \underline{b}^{-1} & \cdots & \underline{b}^{1-n} \\
\underline{a}^{2} & \underline{a}^{2} \underline{b}^{-1} & \cdots & \underline{a}^{2} \underline{b}^{1-n} \\
\underline{a} & \underline{a}^{-1} & \cdots & \underline{b}^{-1} \underline{b}^{1-n}
\end{array}\right] \\
\left.+e^{j\left(\theta_{r}+\delta\right)}\left[\begin{array}{cccc}
1 & \underline{b} & \cdots & \underline{b}^{n-1} \\
1 & \underline{b} & \cdots & \underline{b}^{n-1} \\
1 & \underline{b} & \cdots & \underline{b}^{n-1}
\end{array}\right]\right\}\left[\begin{array}{c}
i_{r 1} \\
\vdots \\
i_{r n}
\end{array}\right]
\end{gathered}
$$

where $\underline{b}$ is the complex number $e^{j \alpha_{r}}$. The mutual stator-rotor flux linkage complex vector $\underline{\boldsymbol{\lambda}}_{s r}$ is obtained by adding all three rows of (15) and multiplying by $2 / 3$, thus

$$
\begin{aligned}
& \underline{\boldsymbol{\lambda}}_{s r}=L_{m}\left\{\left(1+\underline{a}+\underline{a}^{2}\right) e^{-j\left(\theta_{r}+\delta\right)}\left[\begin{array}{llll}
1 & \underline{b}^{-1} & \cdots & \underline{b}^{1-n}
\end{array}\right]\right. \\
& \left.+e^{j\left(\theta_{r}+\delta\right)}\left[\begin{array}{llll}
1 & \underline{b} & \cdots & \underline{b}^{n-1}
\end{array}\right]\right\}\left[\begin{array}{c}
i_{r 1} \\
i_{r 2} \\
\vdots \\
i_{r n}
\end{array}\right]
\end{aligned}
$$

Since $1+\underline{a}+\underline{a}^{2}=0$, the first term on the right-hand side of (16) is always zero, therefore, $\underline{\lambda}_{s r}$ simply becomes

$$
\underline{\boldsymbol{\lambda}}_{s r}=\frac{n}{2} L_{m} e^{j\left(\theta_{r}+\delta\right)} \underline{\mathbf{i}}_{r}
$$

where

$$
\underline{\mathbf{i}}_{r}=\frac{2}{n}\left[\begin{array}{llll}
1 & \underline{b} & \cdots & \underline{b}^{n-1}
\end{array}\right]\left[\begin{array}{c}
i_{r 1} \\
i_{r 2} \\
\vdots \\
i_{r n}
\end{array}\right]
$$

defines the space-vector representation of the rotor currents.

The total stator flux, given by the sum of (3) and (17), is

$$
\underline{\boldsymbol{\lambda}}_{s}=\underline{\boldsymbol{\lambda}}_{s s}+\underline{\boldsymbol{\lambda}}_{s r}=L_{s} \underline{\mathbf{i}}_{s}+\frac{n}{2} L_{m} e^{j\left(\theta_{r}+\delta\right)} \underline{\mathbf{i}}_{r}
$$

where $L_{s}=L_{l s}+\frac{3}{2} L_{m s}$. Note that, in this derivation, no assumption has been made regarding the waveform of the rotor currents, and (19) is valid for any type of excitation, as well as during transient operation.

In space-vector notation, the stator voltage $\underline{\mathbf{v}}_{s}$ is known to be [1]

$$
\underline{\mathbf{v}}_{s}=r_{s} \underline{\mathbf{i}}_{s}+p \underline{\boldsymbol{\lambda}}_{s}
$$

where $r_{s}$ is the stator resistance. Taking the time derivative of (19) and substituting into (20) yields

$$
\underline{\mathbf{v}}_{s}=r_{s} \underline{\mathbf{i}}_{s}+L_{s} p \mathbf{i}_{s}+\frac{n}{2} L_{m} e^{j\left(\theta_{r}+\delta\right)}\left(p+j \omega_{r}\right) \underline{\mathbf{i}}_{r} .
$$

This expression has the same structure as the well-known $d-q$ model available in the literature, the main difference being the phase angle introduced by the complex exponential term, which is due to the arbitrary choice of reference for the magnetic axis of the first rotor loop. Also, since a nonsinusoidal rotor bar distribution is considered, the number of rotor bars appears explicitly.

\section{ROTOR MODEL}

Given the structural symmetry of the rotor, it is convenient to model the cage as $n$ identical magnetically coupled circuits. One particular advantage of this approach is that it is applicable to rotors with a nonintegral number of bars per pole. For simplicity, each loop is defined by two adjacent rotor bars and the connecting portions of the end rings between them.

For the purpose of analysis, each rotor bar and segment of end ring is substituted by an equivalent circuit representing the resistive and inductive nature of the cage [4], [5], [15]. It is also convenient to carry out the analysis using mesh currents as 


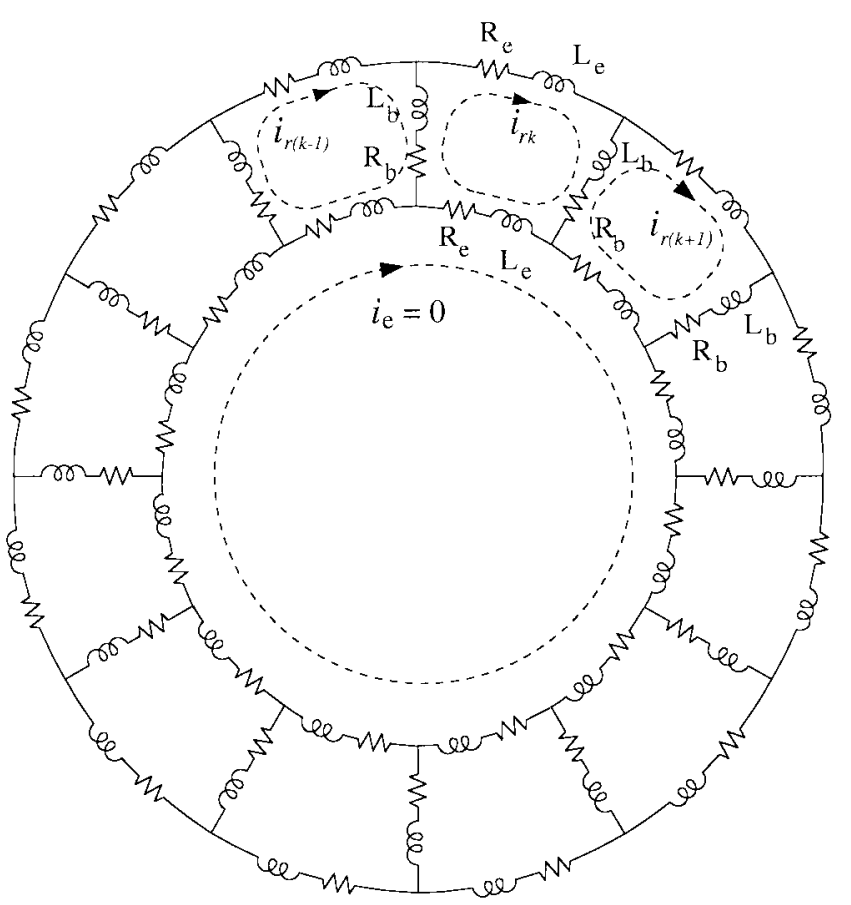

Fig. 3. Rotor cage equivalent circuit.

the independent variables. Such an equivalent circuit is shown in Fig. 3.

In general, there are $n+1$ independent meshes defined by the $n$ rotor loops plus one formed by any one of the end rings. However, in the absence of an axial flux component, the circumferential current in the end ring $i_{e}$ is identically zero, hence, it will not be considered here.

The loop equation for the $k$ th rotor circuit is

$$
0=2\left(R_{b}+R_{e}\right) i_{r k}-R_{b} i_{r(k-1)}-R_{b} i_{r(k+1)}+p \lambda_{r k}
$$

where $R_{b}$ represents the bar resistance, $R_{e}$ is the end-ring segment resistance, $\lambda_{r k}$ is the total flux linked by the $k$ th loop, and $i_{r k}$ is the loop current. Since each loop is assumed to be identical, (22) is valid for every loop, therefore, the rotor voltage equation in matrix form is

$$
\left[\begin{array}{c}
0 \\
0 \\
\vdots \\
0
\end{array}\right]=\left[\begin{array}{cccc}
R_{o} & -R_{b} & \cdots & -R_{b} \\
-R_{b} & R_{o} & \cdots & 0 \\
\vdots & \vdots & \ddots & \vdots \\
-R_{b} & 0 & \cdots & R_{o}
\end{array}\right]\left[\begin{array}{c}
i_{r 1} \\
i_{r 2} \\
\vdots \\
i_{r n}
\end{array}\right]+p\left[\begin{array}{c}
\lambda_{r 1} \\
\lambda_{r 2} \\
\vdots \\
\lambda_{r n}
\end{array}\right]
$$

with $R_{o}=2\left(R_{b}+R_{e}\right)$.

The rotor equation in space-vector form is obtained by applying the transformation given in (18)-(23) yielding

$$
\underline{\mathbf{0}}=r_{r} \underline{\mathbf{i}}_{r}+p \underline{\boldsymbol{\lambda}}_{r}
$$

where $\underline{\mathbf{0}}$ represents the zero voltage vector due to the squirrel cage and $r_{r}$ is the equivalent rotor resistance in the subspace defined by the space-vector transformation.

The total rotor flux $\Lambda_{r}=\left[\begin{array}{llll}\lambda_{r 1} & \lambda_{r 2} & \cdots & \lambda_{r n}\end{array}\right]^{T}$ can be divided into two components, one due to the stator currents, $\Lambda_{r s}$ and one due to the rotor currents $\boldsymbol{\Lambda}_{r r}$. Again, for clarity of explanation, each component will be developed separately.

\section{A. Rotor Flux Due to Stator Currents}

The mutual coupling between rotor and stator is given by

$$
\boldsymbol{\Lambda}_{r s}=\left[\begin{array}{c}
\lambda_{r 1 s} \\
\lambda_{r 2 s} \\
\vdots \\
\lambda_{r n s}
\end{array}\right]=\left[\begin{array}{ccc}
L_{1 a} & L_{1 b} & L_{1 c} \\
L_{2 a} & L_{2 b} & L_{2 c} \\
\vdots & \vdots & \vdots \\
L_{n a} & L_{n b} & L_{n c}
\end{array}\right]\left[\begin{array}{c}
i_{a s} \\
i_{b s} \\
i_{c s}
\end{array}\right]
$$

Because of energy considerations, $L_{i x}=L_{x i}$ for $i=1, \ldots, n$ and for $x=a, b, c$; and they correspond to those defined in Section II-B. Using complex notation and multiplying the rows of (25) by $1, \underline{b}, \ldots, \underline{b}^{n-1}$ yields (26), shown at the bottom of the page. The complex vector $\underline{\boldsymbol{\lambda}}_{r s}$ is obtained by adding all the rows of (26) and multiplying by $2 / n$, thus

$$
\begin{aligned}
\underline{\lambda}_{r s}= & \frac{L_{m}}{n}\left\{n e^{-j\left(\theta_{r}+\delta\right)}\left[\begin{array}{lll}
1 & \underline{a} & \underline{a}^{2}
\end{array}\right]\right. \\
& \left.+e^{j\left(\theta_{r}+\delta\right)}\left(1+\cdots+\underline{b}^{2(n-1)}\right)\left[\begin{array}{lll}
1 & \underline{a}^{2} & \underline{a}
\end{array}\right]\right\}\left[\begin{array}{c}
i_{a s} \\
i_{b s} \\
i_{c s}
\end{array}\right] .
\end{aligned}
$$

Since $1+\underline{b}^{2}+\cdots+\underline{b}^{2(n-1)}=0$ and using the definition of the stator current space vector $\underline{\mathbf{i}}_{s}$, (27) reduces to

$$
\underline{\boldsymbol{\lambda}}_{r s}=\frac{3}{2} L_{m} e^{-j\left(\theta_{r}+\delta\right)} \underline{\mathbf{i}}_{s} .
$$

\section{B. Rotor Flux Due to Rotor Currents}

The total flux linked by the $k$ th rotor circuit and due only to the rotor currents is given by

$$
\begin{aligned}
\lambda_{r k r}=L_{k 1} i_{r 1}+\cdots+L_{k n} i_{r n} & +2\left(L_{e}+L_{b}\right) i_{r k} \\
& -L_{b}\left(i_{r(k-1)}+i_{r(k+1)}\right)
\end{aligned}
$$

where $L_{k i}$ represents the mutual magnetic coupling between rotor loops $k$ and $i$ and $L_{k k}$ is the self-inductance of the $k$ th loop.

Because of the structural symmetry of the rotor, (29) is valid for every loop, hence, $\boldsymbol{\Lambda}_{r r}$ can be written in matrix form as

$$
\left[\begin{array}{c}
\lambda_{r 1 r} \\
\lambda_{r 2 r} \\
\vdots \\
\lambda_{r n r}
\end{array}\right]=\left[\begin{array}{cccc}
L_{11}+L_{o} & L_{12}-L_{b} & \cdots & L_{1 n}-L_{b} \\
L_{21}-L_{b} & L_{22}+L_{o} & \cdots & L_{2 n} \\
\vdots & \vdots & \ddots & \vdots \\
L_{n 1}-L_{b} & L_{n 2} & \cdots & L_{n n}+L_{o}
\end{array}\right]\left[\begin{array}{c}
i_{r 1} \\
i_{r 2} \\
\vdots \\
i_{r n}
\end{array}\right]
$$

$$
\left[\begin{array}{c}
\lambda_{r 1 s} \\
\underline{b} \lambda_{r 2 s} \\
\vdots \\
\underline{b}^{n-1} \lambda_{r n s}
\end{array}\right]=\frac{L_{m}}{2}\left\{e^{-j\left(\theta_{r}+\delta\right)}\left[\begin{array}{ccc}
1 & \underline{a} & \underline{a}^{2} \\
1 & \underline{a} & \underline{a}^{2} \\
\vdots & \vdots & \vdots \\
1 & \underline{a} & \underline{a}^{2}
\end{array}\right]+e^{j\left(\theta_{r}+\delta\right)}\left[\begin{array}{ccc}
1 & \underline{a}^{2} & \underline{a} \\
\underline{b}^{2} & \underline{a}^{2} \underline{b}^{2} & \underline{a}^{2} \\
\vdots & \vdots & \vdots \\
\underline{b}^{2(n-1)} & \underline{a}^{2} \underline{b}^{2(n-1)} & \underline{a} \underline{b}^{2(n-1)}
\end{array}\right]\right\}\left[\begin{array}{c}
i_{a s} \\
i_{b s} \\
i_{c s}
\end{array}\right]
$$


with $L_{o}=2\left(L_{b}+L_{e}\right)$. The self- and mutual inductances defined in (30) are obtained by means of winding functions. The self-inductance of the $k$ th loop is found to be [6]

$$
L_{k k k}=\frac{\mu_{o} l r}{g} \int_{0}^{2 \pi} N_{k}^{2}(\theta) d \theta=\frac{\mu_{o} l r}{g} \alpha_{r}\left(1-\frac{\alpha_{r}}{2 \pi}\right)
$$

and the mutual inductance between the $k$ th and the $i$ th rotor loops is

$$
L_{k i}=\frac{\mu_{o} l r}{g} \int_{0}^{2 \pi} N_{k}(\theta) N_{i}(\theta) d \theta=\frac{\mu_{o} l r}{g}\left(-\frac{\alpha_{r}^{2}}{2 \pi}\right) .
$$

As expected, both $L_{k k}$ and $L_{k i}$ are constants that only depend on the rotor dimensions and not on their relative position. Substituting (31) and (32) into (30) and multiplying each row by $1, \underline{b}, \ldots, \underline{b}^{n-1}$ yields (33), shown at the bottom of the page, where we have used the identity $\underline{b}^{n+m}=\underline{b}^{m}$.

Adding all the rows in (33) and reducing terms, we obtain the rotor flux complex vector due to the rotor currents

$$
\begin{aligned}
\underline{\lambda}_{r r} & =\left(L_{o}+\frac{n}{n-1} L_{k k}-2 L_{b} \cos \alpha_{r}\right) \underline{\mathbf{i}}_{r} \\
& =\underbrace{\left(2 L_{b}\left(1-\cos \alpha_{r}\right)+2 L_{e}+\frac{\mu_{o} l r}{g} \alpha_{r}\right)}_{L_{r}} \underline{\mathbf{i}}_{r} .
\end{aligned}
$$

The proportionality constant $L_{r}$ corresponds to the equivalent rotor inductance. Note that its value is expressed only in terms of rotor dimensions and bar and end-ring inductance values.

The total rotor flux vector, defined as the sum of (28) and (34), is

$$
\underline{\boldsymbol{\lambda}}_{r}=\frac{3}{2} L_{m} e^{-j\left(\theta_{r}+\delta\right)} \underline{\mathbf{i}}_{s}+L_{r} \underline{\mathbf{i}}_{r} .
$$

\section{Rotor Equivalent Resistance}

The equivalent rotor resistance is obtained from the first term on the right-hand side of (23). The procedure is identical to the one used to obtain the rotor flux, therefore, it will not be repeated here. It suffices to say that after multiplying each row of the first term in (23) by $1, \underline{b}, \ldots, \underline{b}^{n-1}$, adding and simplifying it yields

$$
\begin{aligned}
r_{r} & =R_{o}-R_{b}\left(\underline{b}^{n-1}+\underline{b}\right) \\
& =2 R_{e}+2 R_{b}\left(1-\cos \alpha_{r}\right) .
\end{aligned}
$$

Note that equivalent rotor resistance $r_{r}$ is expressed in terms of the actual bar and end ring resistance values, making it straightforward to include changes in their values due to temperature or skin effect.

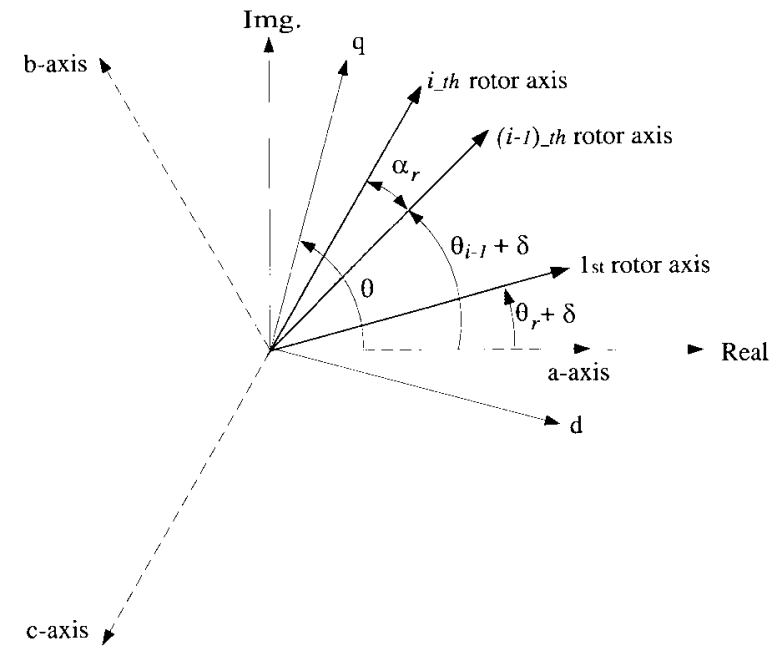

(a)

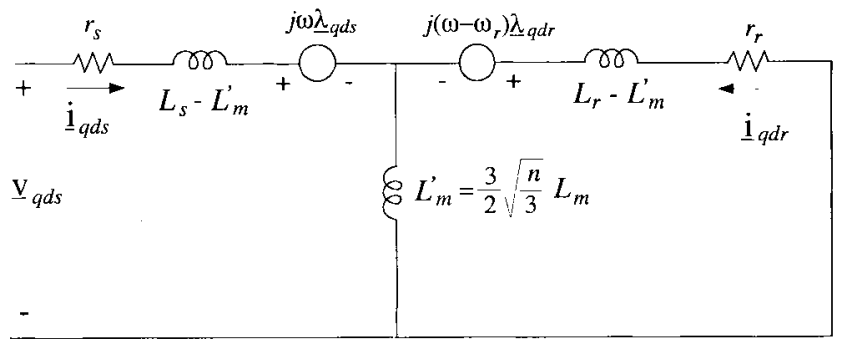

(b)

Fig. 4. (a) Common reference frame definition. (b) Complex vector equivalent circuit.

Finally, substituting (35) into (24) yields the rotor equation in the subspace defined by the rotor space-vector transformation

$$
\underline{\mathbf{0}}=r_{r} \underline{\mathbf{i}}_{r}+\frac{3}{2} L_{m} e^{-j\left(\theta_{r}+\delta\right)}\left(p-j \omega_{r}\right) \underline{\mathbf{i}}_{s}+L_{r} p \underline{\mathbf{i}}_{r} .
$$

Equations (21) and (37) correspond to the complex vector model of the squirrel-cage induction machine and fully define the electrical behavior for both steady-state and transient operation.

\section{Complex Vector EQuivalent Circuit}

It is known that the sinusoidal coupling between the stator and rotor circuits can be eliminated by referring all the equations to a common reference frame [16]. Fig. 4 shows an arbitrary $d-q$ reference frame rotating at angular speed $\omega$. The required $d-q$ variables in this common reference frame

$$
\left[\begin{array}{c}
\lambda_{r 1 r} \\
\underline{b} \lambda_{r 2 r} \\
\vdots \\
\underline{b}^{n-1} \lambda_{r n r}
\end{array}\right]=\left[\begin{array}{cccc}
L_{k k}+L_{o} & \underline{b}^{n-1}\left(L_{k i}-L_{b}\right) & \cdots & \underline{b}\left(L_{k i}-L_{b}\right) \\
\underline{b}\left(L_{k i}-L_{b}\right) & L_{k k}+L_{o} & \cdots & \underline{b}^{2} L_{k i} \\
\vdots & \vdots & \ddots & \vdots \\
\underline{b}^{n-1}\left(L_{k i}-L_{b}\right) & \underline{b}^{n-2} L_{k i} & \cdots & L_{k k}+L_{o}
\end{array}\right] \times\left[\begin{array}{c}
i_{r 1} \\
\underline{b} i_{r 2} \\
\vdots \\
\underline{b}^{n-1} i_{r n}
\end{array}\right]
$$


are defined by the vector transformations

$$
\begin{aligned}
\underline{\mathbf{i}}_{q d s} & =\frac{2}{3} e^{-j \theta} \underline{\mathbf{i}}_{s} \\
\underline{\mathbf{i}}_{q d r} & =\frac{2}{3} \sqrt{\frac{n}{3}} e^{-j\left(\theta-\theta_{r}-\delta\right)} \underline{\mathbf{i}}_{r} .
\end{aligned}
$$

Applying this transformation to (21) and (37) yields

$$
\begin{aligned}
\underline{\mathbf{v}}_{q d s} & =r_{s} \underline{\mathbf{i}}_{q d s}+L_{s} p \underline{\mathbf{i}}_{q d s}+\frac{3}{2} \sqrt{\frac{n}{3}} L_{m} p \underline{\mathbf{i}}_{q d r}+j \omega \underline{\boldsymbol{\lambda}}_{q d s} \\
\underline{\mathbf{0}} & =r_{r} \underline{\mathbf{i}}_{q d r}+L_{r} p \underline{\mathbf{i}}_{q d r}+\frac{3}{2} \sqrt{\frac{n}{3}} L_{m} p \dot{\mathbf{i}}_{q d s}+j\left(\omega-\omega_{r}\right) \underline{\boldsymbol{\lambda}}_{q d r}
\end{aligned}
$$

where

$$
\begin{aligned}
& {\underline{\lambda_{q d s}}}=L_{s} \underline{\mathbf{i}}_{q d s}+\frac{3}{2} \sqrt{\frac{n}{3}} L_{m} \underline{\mathbf{i}}_{q d r} \\
& \underline{\boldsymbol{\lambda}}_{q d r}=\frac{3}{2} \sqrt{\frac{n}{3}} L_{m \underline{\mathbf{i}}_{q d s}}+L_{r} \underline{\mathbf{i}}_{q d r} .
\end{aligned}
$$

The corresponding equivalent circuit representing the machine in an arbitrary rotating reference frame is shown in Fig. 4.

The analysis carried out so far has implicitly assumed a machine having only one pair of poles, the extension to multipole structures is quite straightforward, and it only requires one to substitute mechanical angles by their electrical equivalents.

It is important to point out that, in contrast to the usual full set of differential equations required to solve for rotor bar currents, the use of the space-vector transformation defined in (18) gives a much simpler description of the machine, regardless of the number of rotor bars, thus providing a faster analysis tool.

\section{INVERSE CURRENT TRANSFORMATION}

In general, all $n$ rotor currents need to be mapped into a full $n$-dimensional vector space. This new $n$-dimensional space is defined by the transformation [18]

$$
\left[\begin{array}{c}
\underline{i}_{r 1}^{\prime} \\
\underline{i}_{r 2}^{\prime} \\
\vdots \\
\underline{i}_{r n}^{\prime}
\end{array}\right]=\left[\begin{array}{cccc}
1 & 1 & \cdots & 1 \\
1 & \underline{b} & \cdots & \underline{b}^{n-1} \\
\vdots & \vdots & \ddots & \vdots \\
1 & \underline{b}^{n-1} & \cdots & \underline{b}^{(n-1)(n-1)}
\end{array}\right]\left[\begin{array}{c}
i_{r 1} \\
i_{r 2} \\
\vdots \\
i_{r n}
\end{array}\right]
$$

where $\underline{i}_{r i}^{\prime}$ represents the $i$ th rotor current in the new space.

Upon applying (43) to the voltage equations of a symmetrical squirrel-cage machine results in [17], [18]

$$
\mathbf{0}=\mathbf{R}_{r}^{\prime} \mathbf{i}_{r}^{\prime}+\mathbf{L}_{r}^{\prime} p\left(\dot{\mathbf{i}}_{r}^{\prime}\right)+\mathbf{T} \mathbf{L}_{r s} p\left(\mathbf{i}_{s}\right)+\mathbf{T} p\left(\mathbf{L}_{r s}\right) \mathbf{i}_{s}
$$

where $\mathbf{i}_{r}^{\prime}=\mathbf{T} \mathbf{i}_{r}, \mathbf{i}_{s}^{\prime}=\mathbf{T i}_{s}, \mathbf{R}_{r}^{\prime}=\mathbf{T} \mathbf{R}_{r} \mathbf{T}^{-1}, \mathbf{L}_{r}^{\prime}=\mathbf{T} \mathbf{L}_{r} \mathbf{T}^{-1}$, and $\mathbf{T}$ is the transformation matrix (43).

If, in addition, the stator currents are also mapped into a new vector space defined by a transformation $\mathbf{T}_{s}$, similar to $\mathbf{T}$ but substituting $\underline{b}$ by $\underline{a}=e^{j 2 \pi / 3}$ and $n$ by 3 , results in

$$
\mathbf{0}=\mathbf{R}_{r}^{\prime} \mathbf{i}_{r}^{\prime}+\mathbf{L}_{r}^{\prime} p\left(\mathbf{i}_{r}^{\prime}\right)+\mathbf{L}_{r s}^{\prime} p\left(\mathbf{i}_{s}^{\prime}\right)+p\left(\mathbf{L}_{r s}^{\prime}\right) \mathbf{i}_{s}^{\prime}
$$

where $\mathbf{i}_{s}^{\prime}=\mathbf{T}_{s} \mathbf{i}_{s}$ and

$$
\mathbf{L}_{r s}^{\prime}=\mathbf{T L}_{r s} \mathbf{T}_{s}^{-1}=\left[\begin{array}{ccc}
0 & 0 & 0 \\
0 & L & 0 \\
0 & 0 & 0 \\
\vdots & \vdots & \vdots \\
0 & 0 & L^{*}
\end{array}\right]
$$

where the superscript $*$ denotes complex conjugate. Since the input voltage vector in (45) is identically zero, the only source of excitation comes from the mutual rotor-stator coupling terms. Given the structure of $\mathbf{L}_{r s}^{\prime}$, regardless of the instantaneous value of the stator currents, only the second and $n$th rows of (45) will yield a nonzero rotor current [17]. The resultant differential equations are of the form

$$
0=R_{r}^{\prime} i_{r k}^{\prime}+L_{r}^{\prime} \frac{d i_{r k}^{\prime}}{d t}
$$

for $k \neq 2$ and $n$. Their solutions are independent of the stator currents and correspond to a trivial case (e.g., identically zero for all time), therefore, they can be eliminated from the solution set. On the other hand, the equations corresponding to the second and last rows of (45) are

$$
\begin{aligned}
& 0=R_{r}^{\prime} i_{r 2}^{\prime}+L_{r}^{\prime} \frac{d i_{r 2}^{\prime}}{d t}+L \frac{d i_{s 1}^{\prime}}{d t}+i_{s 1}^{\prime} \frac{d L}{d t} \\
& 0=R_{r}^{\prime} i_{r n}^{\prime}+L_{r}^{\prime} \frac{d i_{r n}^{\prime}}{d t}+L^{*} \frac{d i_{s 2}^{\prime}}{d t}+i_{s 2}^{\prime} \frac{d L^{*}}{d t}
\end{aligned}
$$

and their solutions depend on the instantaneous stator currents $i_{s 1}^{\prime}$ and $i_{s 2}^{\prime}$. From here, it follows that the inverse current relation can always be written simply as

$$
\left[\begin{array}{c}
i_{r 1} \\
i_{r 2} \\
i_{r 3} \\
\vdots \\
i_{r n}
\end{array}\right]=T^{-1}\left[\begin{array}{c}
0 \\
i_{r 2}^{\prime} \\
0 \\
\vdots \\
i_{r n}^{\prime}
\end{array}\right]
$$

Thus, due to lack of excitation, only the second and last column of the inverse transformation matrix are of interest. Furthermore, from (43), it is clear that $i_{r 2}^{\prime}$ corresponds to the space-vector definition given in (18) and $i_{r n}^{\prime}$ is the complex conjugate. Hence, taking the inverse transformation and substituting yields

$$
\left[\begin{array}{c}
i_{r 1} \\
i_{r 2} \\
i_{r 3} \\
\vdots \\
i_{r n}
\end{array}\right]=\frac{1}{n}\left[\begin{array}{cc}
1 & 1 \\
\underline{b}^{-1} & \underline{b}^{1-n} \\
\vdots & \underline{b}^{2(1-n)} \\
\underline{b}^{1-n} & \underline{b}^{-(1-n)^{2}}
\end{array}\right]\left[\begin{array}{c}
\underline{\mathbf{i}}_{r} \\
\underline{\mathbf{i}}_{r}^{*}
\end{array}\right] .
$$

Equation (50) shows that, for a symmetrical squirrel-cage machine, only the complex conjugate subspaces are externally excited through the stator coupling, while the rest can never be excited. Therefore the original $n$-dimensional space can be fully represented by the bidimensional subspace spanned by (18). Finally, the inverse transformation is simply

$$
\begin{aligned}
i_{r i} & =\frac{2}{n} \operatorname{Re}\left\{\underline{b}^{-(i-1)} \underline{\mathbf{i}}_{r}\right\} \\
& =\frac{2}{n}\left[i_{x r} \cos (i-1) \alpha_{r}+i_{y r} \sin (i-1) \alpha_{r}\right]
\end{aligned}
$$


where Re represents the real part and $i_{x r}$ and $i_{y r}$ are the real and imaginary components of the complex vector $\underline{\underline{i}}_{r}$. Note that this result is completely general and it does not assume any symmetry in the rotor current distribution.

\section{Electromagnetic TORQue}

Neglecting saturation, the electromagnetic torque can be expressed as the partial variation of the coenergy with respect to rotor position [1], [6]

$$
T_{e}=\mathbf{i}_{s}^{T} \frac{\partial \mathbf{L}_{s r}}{\partial \theta_{r}} \mathbf{i}_{r}
$$

where $\mathbf{i}_{r}=\left[\begin{array}{llll}i_{r 1} & i_{r 2} & \cdots & i_{r n}\end{array}\right]^{T}$ and $\mathbf{L}_{s r}$ corresponds to the rotor-stator mutual inductance matrix. After some matrix manipulation, the mutual inductance can be written as

$$
\begin{aligned}
\mathbf{L}_{s r}=\frac{L_{m}}{2} & \left\{e^{j\left(\theta_{r}+\delta\right)}\left[\begin{array}{c}
1 \\
\underline{a}^{2} \\
\underline{a}
\end{array}\right]\left[\begin{array}{llll}
1 & \underline{b} & \cdots & \underline{b}^{n-1}
\end{array}\right]\right. \\
& \left.+e^{-j\left(\theta_{r}+\delta\right)}\left[\begin{array}{c}
1 \\
\underline{a} \\
\underline{a}^{2}
\end{array}\right]\left[\begin{array}{llll}
1 & \underline{b}^{-1} & \cdots & \underline{b}^{1-n}
\end{array}\right]\right\} .
\end{aligned}
$$

Note that only the exponential terms depend on the rotor position, hence, the partial differentiation is quite easily obtained. After differentiating and premultiplying by $\mathbf{i}_{s}^{T}$ and postmultiplying by $\mathbf{i}_{r}$ yields

$$
T_{e}=-\left(\frac{3}{2}\right)\left(\frac{n}{2}\right) L_{m} \operatorname{Im}\left\{e^{j\left(\theta_{r}+\delta\right)} \underline{\mathbf{i}}_{s}^{*} \underline{\mathbf{i}}_{r}\right\}
$$

where $\mathrm{Im}$ represents the imaginary part.

It is important to point out that (54) is similar to the usual expression derived for wound-rotor machines. The term $n / 2$ arises from the arbitrary constant in the definition of the rotor current space vector and the inductance term $L_{m}$ includes the equivalent of the turns ratio found in the usual model.

\section{Simulation RESUlts}

To validate the proposed complex vector model, a set of simulations was prepared. The main objective of the simulations is to compare the results of the proposed model to those of the full matrix model of the induction machine. The full matrix model has been well documented in the literature, and its correctness has been proven over the years. The most recent examples can be found in [8], [15]. For simulation purposes, a typical 5-hp four-pole machine was used. The results from the full matrix model and the complex vector model are shown superimposed in Figs. 5 and 6 for sinusoidal excitation. Fig. 7 shows the results for nonsinusoidal excitation. The agreement in all cases is perfect. The computation times required in each case are shown in Table I. The reduction in computational time achieved by the complex vector model is overwhelming. Note that both models fully predict the rotor bar and endring currents. However, at high speeds, the full matrix model is prone to larger numerical errors due to the multiple matrix
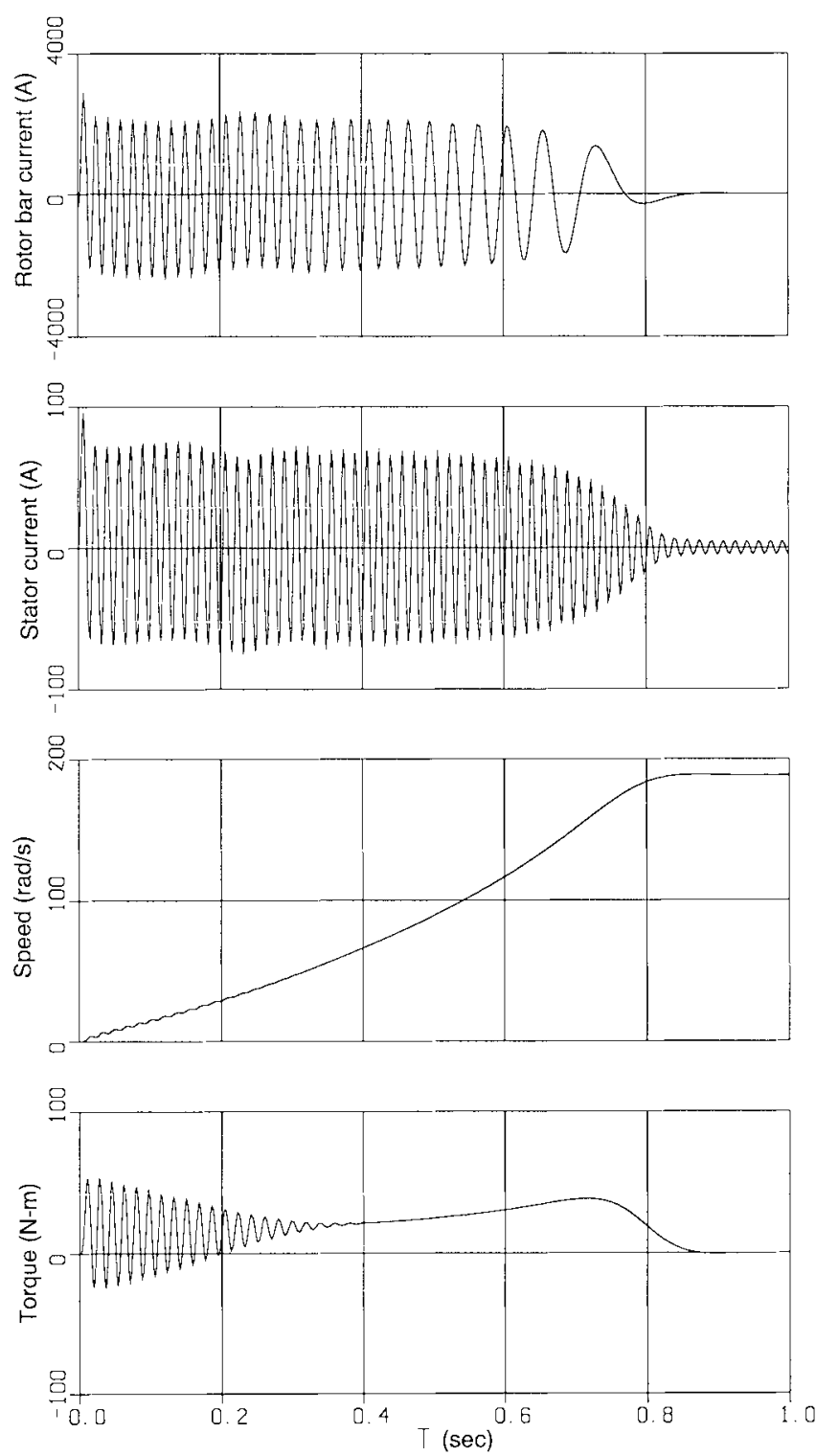

Fig. 5. Complex vector and full matrix model results (superimposed). Rotor bar current, stator current, rotor speed, and electromagnetic torque during free acceleration.

inversions combined to the rapid change in mutual inductance. Decreasing the integration step helps minimize this error, however, this increases the computation time even more. The complex vector model, on the other hand, is free of such problems.

\section{Three-Phase EQuivalent Parameters}

Another important advantage of the complex vector model is its ability to predict the parameters of an equivalent woundrotor machine. The equivalent parameters are most easily obtained by introducing a slight modification to the rotor current space-vector definition

$$
\underline{\mathbf{i}}_{r}^{\prime}=\frac{n}{3} \frac{L_{m}}{L_{m s}} e^{j \delta} \underline{\mathbf{i}}_{r} .
$$



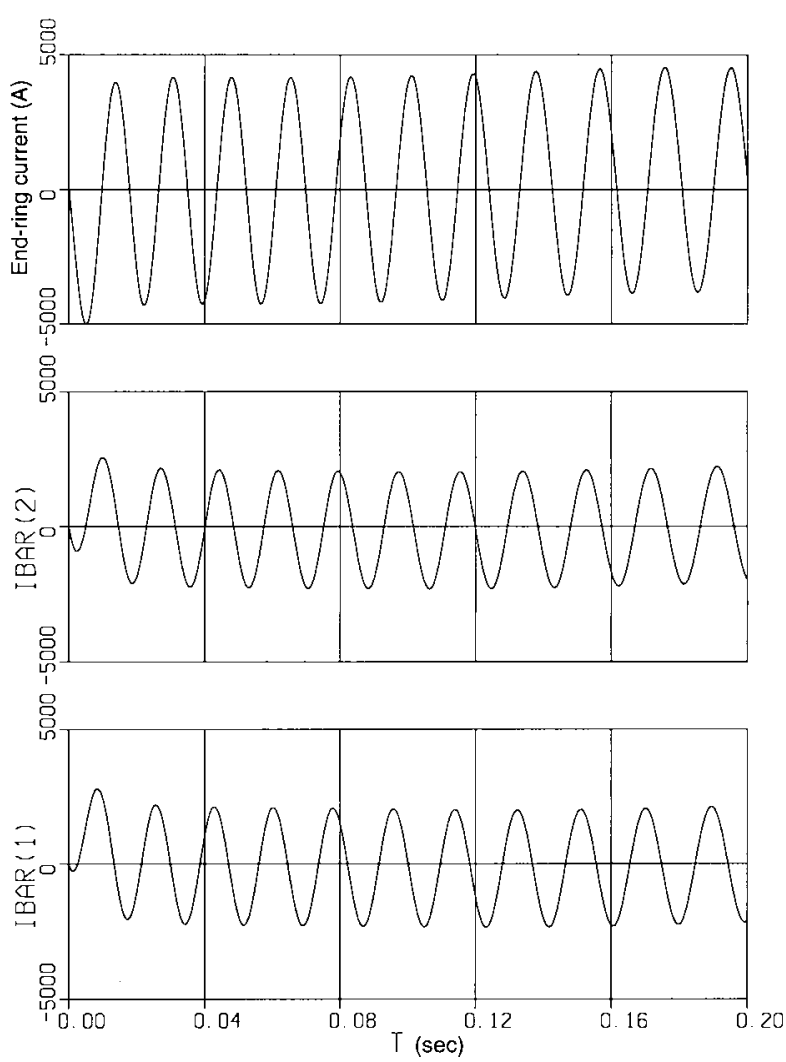

Fig. 6. Complex vector and full matrix model results (superimposed). Rotor currents in two adjacent rotor bars and in the segment of end-ring between them during the initial transient.
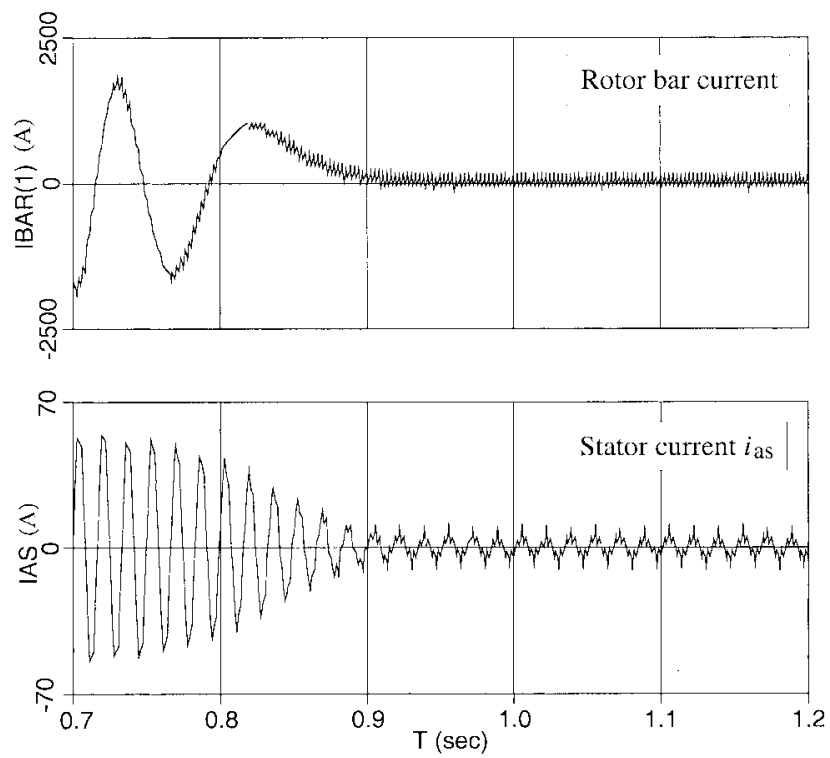

Fig. 7. Complex vector and full matrix model results (superimposed). Stator and rotor bar currents for nonsinusoidal excitation (six step).

Substituting $\underline{\mathbf{i}}_{r}^{\prime}$ into (21) and (37) and comparing to the equations of a sinusoidally wound-rotor induction machine leads to the equivalent stator referred rotor resistance

$$
r_{r}^{\prime}=\left(\frac{3 \pi^{2}}{8}\right) \frac{N_{s}^{2}}{n \sin ^{2} \delta}\left[R_{b}\left(1-\cos \alpha_{r}\right)+R_{e}\right]
$$

TABLE I

Real Time Required to Run Simulations

\begin{tabular}{ccc}
\hline \hline Simulated time & \multicolumn{2}{c}{ Computation time } \\
& Full Matrix & Complex Vector \\
\cline { 2 - 3 } $1 \mathrm{sec}$ & $498 \mathrm{sec}$ & $3 \mathrm{sec}$ \\
\hline
\end{tabular}

TABLE II

LeAKage Inductance Calculations

\begin{tabular}{|c|c|c|c|}
\hline \multicolumn{2}{|c|}{24 bars } & \multicolumn{2}{|c|}{48 bars } \\
\hline Ref. [2] & Comp. vector & Ref. [2] & Comp. vector \\
\hline $2.824 \mathrm{mH}$ & $4.000 \mathrm{mH}$ & $2.510 \mathrm{mH}$ & $2.801 \mathrm{mH}$ \\
\hline
\end{tabular}
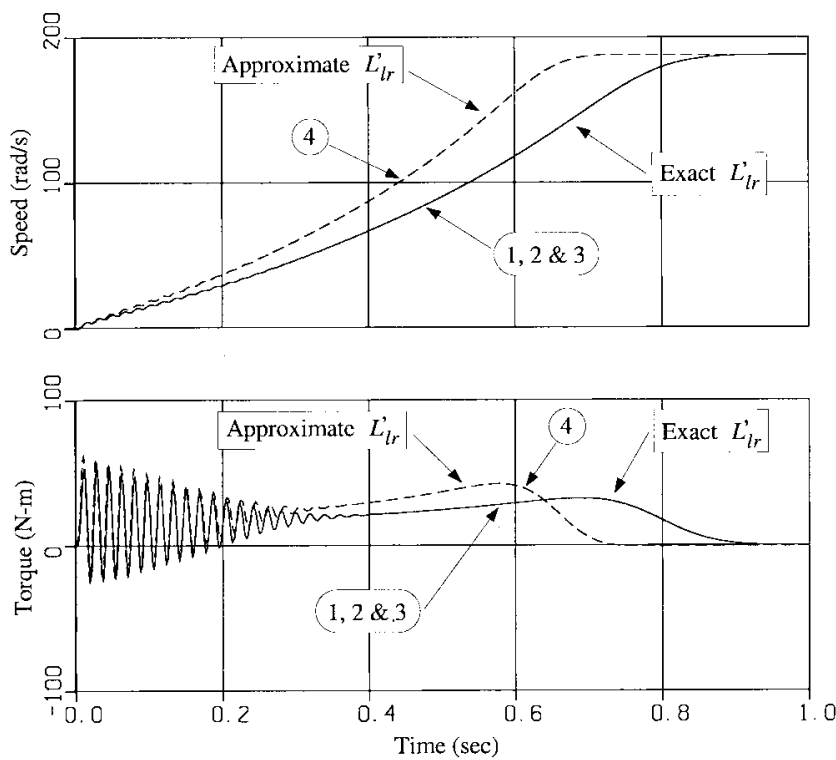

Fig. 8. Change in machine dynamics with rotor leakage inductance (24 rotor bars). 1: Full matrix model. 2: Space-vector model. 3: $d-q$ model using equivalent parameters derived from space-vector model. $4: d-q$ model using equivalent parameters derived from traditional harmonic analysis [2].

and the equivalent rotor leakage inductance

$$
L_{l r}^{\prime}=\frac{6}{n}\left(\frac{\pi}{4}\right)^{2} N_{s}^{2}\left[2 L_{b}+\frac{L_{e}}{\sin ^{2} \delta}\right]+\frac{3}{2} L_{m s}\left[\frac{\delta^{2}}{\sin ^{2} \delta}-1\right] .
$$

Alternate forms to obtain the equivalent rotor parameters of a squirrel-cage machine are presented in [2] and [4]. The technique used is based on harmonic analysis, and to obtain a closed-form solution for the rotor leakage flux, some approximations need to be made. Specifically, some of the spatial harmonic components produced by the rotor fundamental time harmonic component are neglected. This yields an approximate value of equivalent leakage inductance given by

$$
L_{l r}^{\prime} \approx \frac{6}{n}\left(\frac{\pi}{4}\right)^{2} N_{s}^{2}\left[2 L_{b}+\frac{L_{e}}{\sin ^{2} \delta}\right]+\frac{3}{4} L_{m s}\left[\frac{\delta^{2}}{\sin ^{2} \delta}-1\right] .
$$


On the other hand, the complex vector model is based on winding functions which take into account all the harmonic components at once. Also, the novel expression for the leakage inductance derived here has the virtue of simplicity without using the concepts of differential and zig-zag leakages.

The difference between the exact value of $L_{l r}^{\prime}(57)$ and the approximate one (58) is the coefficient of the last term with the exact expression being twice as large. The relative importance of the error depends on the expression between the square brackets. When the number of rotor bars is large, $\delta \rightarrow 0$ and the error is small. Conversely, for rotors with fewer bars, the error may be significant. As an example, the computations for rotors having 24 and 48 bars are presented in Table II.

The effect of using the approximate value of leakage inductance on the torque and speed curves for the 5-hp machine used in the simulation is shown in Fig. 8. In this figure, the dotted line corresponds to the results of the $d-q$ model with $L_{l r}^{\prime}$ computed using (58), while the solid line corresponds to the superimposed solutions of the full matrix model, the complex vector model, and the $d-q$ model with $L_{l r}$ computed using (57).

\section{CONCLUSION}

A detailed mathematical model of a squirrel-cage induction machine has been developed. It has been shown that the use of complex space vectors leads to a simple $d-q$ model without giving up any information regarding the actual rotor bar and end-ring currents. Note that this is true during both transient and steady-state behavior, and it does not assume sinusoidal currents.

The number of equations needed to fully represent the machine is independent of the number of rotor bars considered and the resulting equations have a similar structure to the one found in the usual $d-q$ model. Because of the minimum number of equations used in the final model, the computation time required to simulate the machine is drastically reduced. The three-phase equivalent rotor parameters in terms of actual rotor bar and end-ring resistance and inductance values are also obtained.

\section{REFERENCES}

[1] D. W. Novotny and T. A. Lipo, Vector Control and Dynamics of AC Drives. Oxford, U.K.: Clarendon, 1996.

[2] A. S. Langsdorf, Theory of Alternating Current Machinery, 2nd ed. New York: McGraw-Hill, 1955

[3] P. L. Alger, Induction Machines, 2nd ed. New York: Gordon and Breach, 1970

[4] T. A. Lipo, Introduction to AC Machine Design. Madison, WI: Univ. of Wisconsin Press, 1996.

[5] A. K. Wallace and A. Wright, "Novel simulation of cage windings based on mesh circuit model," IEEE Trans. Power App. Syst., vol. PAS-93, pp. 377-382, Jan./Feb. 1974
[6] H. A. Toliyat, "Analysis of concentrated winding induction and reluctance machines for adjustable speed drive applications," Ph.D. dissertation, Dep. Elect. Comput. Eng., Univ. Wisconsin, Madison, 1991.

[7] H. R. Fudeh and C. M. Ong, "Modeling and analysis of induction machines containing space harmonics," IEEE Trans. Power App. Syst., vol. PAS-102, pts. 1-3, pp. 2608-2628, Aug. 1983.

[8] X. Luo, Y. Liao, H. A. Toliyat, A. El-Antably, and T. A. Lipo, "Multiple couple circuit modeling of induction machines," IEEE Trans. Ind. Applicat., vol. 31, pp. 311-317, Mar./Apr. 1995.

[9] A. K. Wallace, R. Spée, and H. K. Lauw, "Dynamic modeling of brushless doubly-fed machines," in Conf. Rec. IEEE-IAS Annu. Meeting, San Diego CA, 1989, pp. 329-334.

[10] J. Stepina, "Matrix calculation of inductances for the general theory of electrical machines," Elect. Mach. and Power Syst., vol. 11, pp. 53-63, 1986.

[11] S. Williamson and R. C. Healey, "Space vector representation of advanced motor models for vector controlled induction motors," Proc. Inst. Elect. Eng.-Elect. Power Applicat., vol. 143, no. 1, pp. 69-77, 1996.

[12] S. A. Nasar, "Electromechanical energy conversion in nm-winding double cylindrical structures in presence of space harmonics," IEEE Trans. Power App. Syst., vol. PAS-87, pp. 1099-1106, Apr. 1968.

[13] T. A. Lipo, "Theory and control of synchronous machines," ECE 511 Class Notes, Elect. Comput. Eng. Dep., Univ. Wisconsin, Madison, 1991.

[14] N. L. Schmitz and D. W. Novotny, Introductory Electromechanics. New York: Ronald, 1965

[15] H. A. Toliyat and T. A. Lipo, "Transient analysis of cage induction machines under stator, rotor bar, and end ring faults," IEEE Trans. Energy Conv., vol. 10, pp. 241-247, June 1995.

[16] P. Krause and C. Thomas, "Simulation of symmetrical induction machinery," IEEE Trans. Power App. Syst., vol. PAS-84, pp. 1038-1053, Nov. 1965.

[17] A. Muñoz-García, "Analysis and control of a dual stator winding squirrel cage induction machine for high performance drives," preliminary Ph.D. dissertation proposal, Univ. Wisconsin, Madison, 1997.

[18] D. C. White and H. H. Woodson, Electromechanical Energy Conversion. New York: Wiley, 1959.

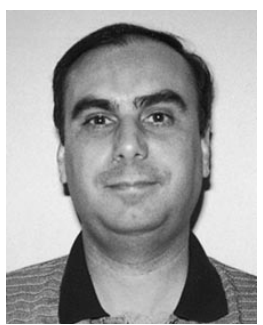

Alfredo R. Muñoz was born in Valparaíso, Chile. $\mathrm{He}$ received the B.S. degree in electrical engineering in 1981 from the Technical University Santa María, Valparaíso, Chile, and the M.S. degree in electrical engineering in 1995 from the University of Wisconsin, Madison, where he is currently working toward the Ph.D. degree.

From 1981 to 1986, he was with Schlumberger Overseas. In 1987, he became a full-time Lecturer and, in 1989, an Assistant Professor in the Electrical Engineering Department, Technical University Santa María. Since 1993, he has been on leave as a Research Assistant at the University of Wisconsin, Madison. He has been involved in several research projects in the areas of variable-frequency drives and power electronics. He was a Fulbright Fellow from 1993 to 1995. His main research interests include electric machines, ac drives, and power electronics.

Thomas A. Lipo (M'64-SM'71-F'87), for a photograph and biography, see p. 486 of the March/April 1999 issue of this TRANSACtIONS. 